acrylamide gel electrophoresis: use of aqueous isopropanol to remove detergent from gels. Anal. Biochem. 120:267-275.

4.Coburn, G.A. and G.A. Mackie. 1999. Degradation of mRNA in Escherichia coli: an old problem with some new twists. Prog. Nucleic Acid Res. Mol. Biol. 62:55-108.

5.D'Alessio, G. and J.F. Riordan. 1997. Ribonucleases: Structures and Functions. Academic Press, New York.

6.Hemsley, A., N. Arnheim, M.D. Toney, G. Cortopassi, and D.J. Galas. 1989. A simple method for site-directed mutagenesis using the polymerase chain reaction. Nucleic Acids Res. 17:6545-6551.

7.Liao, Y.D. 1992. A pyrimidine-guanine sequnce-specific ribonuclease from Rana catesbeiana (bullfrog) oocyte. Nucleic Acids Res. 20:1371-1377.

8.Liao, Y.-D. and J.-J. Wang. 1994. Yolk granules are the major compartment for bullfrog (Rana catesbeiana) oocyte-specific ribonuclease. Eur. J. Biochem. 222:215-220.

9.McDowall, K.J. and S.N. Cohen. 1996. The $\mathrm{N}$-terminal domain of the rne gene product has RNase $\mathrm{E}$ activity and is non-overlapping with the arginine-rich RNA-binding site. J. Mol. Biol. 255:349-355.

10.Smith, B.J. 1996. Chemical cleavage of proteins at cysteinyl residues, p. 385-387. In J.M. Walker (Ed.), The Protein Protocols Handbook. Humana Press, Totowa, NJ.

11.Tao, R., H. Yamane, H. Sassa, H. Mori, T.M. Gradziel, A.M. Dandekar, and A. Sugiura. 1997. Identification of stylar RNases associated with gametophytic self-incompatibility in almond (Prunus dulcis) Plant Cell Physiol. 38:304-311.

12. Wennborg, A., B. Sohlberg, D. Angerer, G. Klein, and A. von Gabain. 1995. A human RNase E-like activity that cleaves RNA sequences involved in mRNA stability control. Proc. Natl. Acad. Sci. USA 92:7322-7326.

We thank Kenneth McDowall for his helpful comments on this manuscript. V.K. was supported by Spezialforschungsbereich grant no. F1707 from the Austrian Science Foundation. Address correspondence to Dr. Vladimir R. Kaberdin and Alexander von Gabain, Institute of Microbiology and Genetics, Vienna Biocenter, Dr. Bohrgasse 9, A-1030, Vienna, Austria. e-mail: vladimir @ gem.univie.ac.at

Received 18 January 2002; accepted 12 April 2002.

Rune-Pär Nilsson', Alexander
von Gabain ${ }^{1,2}$, and Vladimir
R. Kaberdin'1
${ }^{1}$ Vienna Biocenter
${ }^{2}$ InterCell GmbH
Vienna, Austria

Rune-Pär Nilsson', Alexander von Gabain ${ }^{1,2}$, and Vladimir R. Kaberdin Center Vienna, Austria

\section{Sequencing of Difficult Templates Containing Poly(A/T) Tracts: Closure of Sequence Gaps}

\section{BioTechniques 33:276-280 (August 2002)}

A comprehensive working draft of the human genome sequencing project is now available on public databases (4). Much of this sequence is released into the public domain as unfinished sequences in the form of small unaligned contigs, which are then updated regularly until they have reached an accuracy of greater than $99.9 \%$ (1). However, numerous gaps remain in the sequence data (http://www.ensembl.org). Indeed, more than 80 such gaps can be found in a $2-\mathrm{Mb}$ portion of chromosome $17 \mathrm{q} 25$, between the microsatellite markers D17S785 and D17S802, which is im plicated in the development of familial and sporadic forms of esophageal cancer, breast cancer, and ovarian cancer (2,3,7-9). Throughout the progress of sequencing in this area, it has been obvious that we have been able to align and combine fragments from three unfinished clones in our region with greater accuracy than the Human Genome Project simply by using known and new physical mapping data, including unpublished clone-end sequence-tagged sites. Indeed, until recently the sequence maps placed clone 318A15 completely within clone 666A8 (http://ensembl.org and http:// genome.cse.ucsc.edu), but this was not corroborated by physical mapping data with a depth of at least eight clones, which places the whole of 666A8 proximal to $318 \mathrm{~A} 15(5,7)$. Gap closure is not a priority at high-throughput sequencing centers until the clone sequence has reached full draft coverage. One clone, 666A8 (GenBank ${ }^{\circledR}$ no. AC015802) from chromosome 17, demonstrates this apparent problem because it remains in two pieces even after several updates over the past year that changed the status of sequencing from phase two (ordered contigs of draft sequence) to activefin (start of directed sequencing).

It is therefore important that smaller research groups with a vested interest in a particular region of the genome try to complete their own sequence to facilitate the Human Genome Project and further their own research. An example of such a region is the 500-kb tylosis with esophageal cancer gene region on chromosome 17q25, where six gaps have been identified in the sequence data. Closer examination revealed that five of the six gaps were either flanked by sequences containing runs of As or sequences that were very AT rich. The length of these regions varied at each gap (9-28 bp) and was positioned 100-1000 bp from the ends of the known sequences. PCR across two of these gaps indicated that the missing segment was less than $100 \mathrm{bp}$ in each case (Figure 1). However, gap closure via sequencing these PCR products proved unsuccessful and appeared to be impeded by the A runs. Several troubleshooting methods were applied, including the addition of DMSO to the sequencing reactions, an AT-rich cycle protocol and GC-rich cycle protocol, but none of these produced any novel sequence. The hypothesis that the sequencing of these regions is impeded by A runs was strengthened further by the analysis of a gene exon within the tylosis esophageal cancer region that was also flanked on both sides by poly(A) tracts. Only a small amount of good quality sequence $(>99 \%$ correct base calling) was obtained from initial sequencing reactions, and this quickly deteriorated, becoming uninterpretable (Figure 2A). When the data were com pared with the original entry in the National Center for Biotechnology Information database (http://ncbi.nlm. nih.gov), it was found that the sequence deteriorated in both directions after reaching runs of As. New sequencing primers were designed to avoid the As by annealing beyond those regions or to include some of the A run within the $5^{\prime}$ portion of the primer sequence. Sequencing reactions carried out using these new primers produced excellent sequence with greater than $99 \%$ correct base calling that covered the exon and then deteriorated again when the A region on the far side of the exon was reached (Figure 2B).

The technique of designing primers adjacent to or containing part of the problematic poly $(\mathrm{A})$ or $(\mathrm{T})$ tracts was 
then used to obtain sequence across two sequence gaps within the tylosis esophageal cancer region; one of them was the clone 666A8 gap. The sequence data immediately adjacent to these breaks in the contig showed a run of 26 As positioned 299 bp upstream of gap 1 , and a run of 15 Ts was observed 74 bp downstream of gap 1 (Figure 1A). In addition, 19 adjacent As were observed $49 \mathrm{bp}$ upstream of gap 2, and the $100 \mathrm{bp}$ immediately downstream of gap 2 were 74\% AT rich (Figure 1B). Sequencing primers $(3 \mathrm{~B}, 1 \mathrm{~B}, 1 \mathrm{~A}, \mathrm{~S} 5$, and $\mathrm{S} 4)$ were designed that overlapped or primed beclone and human genomic DNA were PCR-amplified using Elongase ${ }^{\circledR}$ Enzyme Mixture (Invitrogen, Carlsbad, for sequencing, according to the manufacturer's instructions. PCR primers and IRD-700 labeled sequencing primers yond these regions (Figure 1). The BAC CA, USA) to produce a $1-\mathrm{kb}$ template

were designed using the Primer3 Program available on the Whitehead Institute Web site (http://www-genome.wi. mit.edu/cgi-bin/ primer/primer3.cgi) and synthesized by MWG-Biotech AG (Ebersberg, Germany). Primer sequences and annealing temperatures are available on request. The PCR cycle included an initial heat denaturation step of $94^{\circ} \mathrm{C}$ for $4 \mathrm{~min}$, followed by $30 \mathrm{cy}-$ cles of $94^{\circ} \mathrm{C}$ for $1 \mathrm{~min}, 1 \mathrm{~min}$ at annealing temperature, and a final extension step of $72^{\circ} \mathrm{C}$ for $5 \mathrm{~min}$. Five microliters of PCR product were assessed on a $1.5 \%$ agarose gel to ensure that a single product of the correct size had been produced. PCR products were cleaned using a QIAquick ${ }^{\mathrm{TM}}$ Gel Extraction Column (Qiagen, Valencia, CA, USA). PCR templates were sequenced using Sequitherm Excel ${ }^{\mathrm{TM}}$ II DNA sequencing kit-LC (for 25-41 cm gels) (Microzone, Lewes, UK). The reactions were

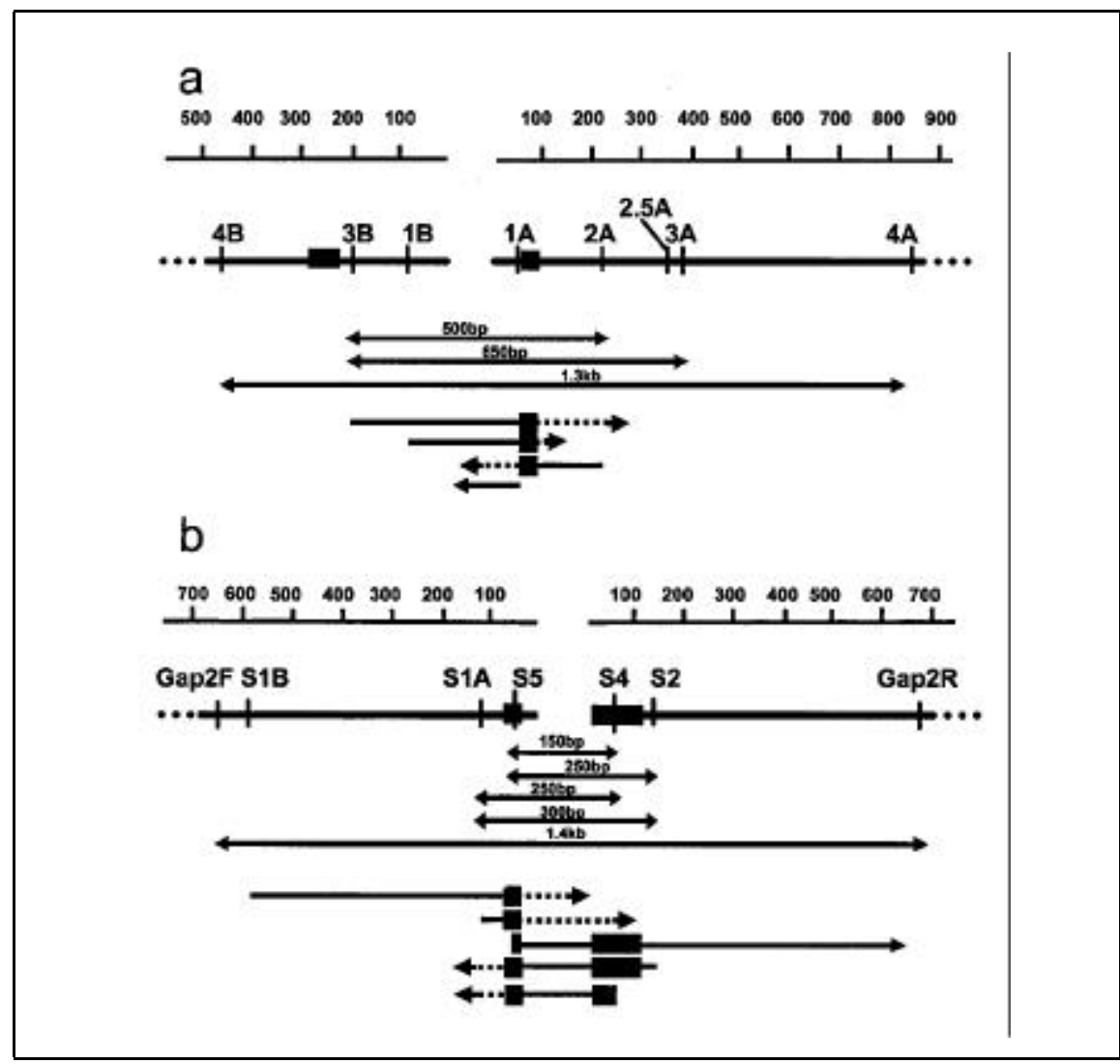

Figure 1. PCR and sequence coverage of two sequence gaps. (A) Gap 1 (between clone 666A8 fragments 2 and 1). (B) Gap 2 (between clone 234P2 fragments 7 and 10). Double-ended arrows, PCR fragments with approximate sizes determined by agarose gel electorphoresis written above; boxes, runs of single A or T bases or AT-rich region (see text for details); and single-ended arrows, sequence data obtained in the direction of the arrow. Solid line represents high-quality data (>99\% correct base calling), and dotted line represents low-quality data (50\% correct base calling by program; $80 \%$ correct base calling by eye; and two or more peaks for $>80 \%$ of bases). Scale bars are in bp. heated for $5 \mathrm{~min}$ at $95^{\circ} \mathrm{C}$ to denature the template, and then $30 \mathrm{PCR}$ cycles of 30 $\mathrm{s}$ at $95^{\circ} \mathrm{C}, 30 \mathrm{~s}$ at annealing temperature, and $1 \mathrm{~min}$ at $70^{\circ} \mathrm{C}$ were performed. Three microliters of stop/loading buffer were added to each reaction and then heated to $75^{\circ} \mathrm{C}$ for $5 \mathrm{~min}$. One microliter was loaded onto a $6 \%$ gel containing urea and formamide for analysis with a DNA sequencer (model 4200; LI-COR, Lincoln, NE, USA) at $2000 \mathrm{~V}$ and $45^{\circ} \mathrm{C}$ for approximately $4 \mathrm{~h}$. These primers successfully produced sequence data in both directions that crossed the gaps, went through the opposite A- or AT-rich tract, and proceeded into the known sequence of the clone (Figure 1). The sequence data obtained beyond each poly(A) or AT-rich tract were relatively poor because of strand slippage, showing two or more peaks at over $80 \%$ of the bases, and being readable by eye for only 50-100 bases. However, it was sufficiently similar to that observed from sequencing reactions in the other direction for these to be identified as the same. In addition, these sequence data were of a quality that would allow the selection of new sequencing primers, even if previous data from this region were unavailable. Identical results were obtained from the BAC clone and total human genomic template, and the experiments were repeated six times. Additional sequencing primers (2.5A, S1B, S1A, and S2) were used to confirm that overlapping data beyond the poly(A) tract were identical to that in the database entry for the clone in question (Figure 1). The GenBank accession numbers for the two novel sequences obtained using this method are AY042936 (gap 1) and AY042219 (gap 2).

We have shown that runs of $\mathrm{A}$ or $\mathrm{T}$ bases can impede DNA polymerases during sequencing reactions and cause slippage and deterioration of the sequence data. Sequencing primers designed to anneal specifically after or at the end of these problematic regions are able to overcome this problem and produce good-quality sequence. This technique has been used to close two longstanding sequencing gaps in a region of chromosome $17 q 25$ that may harbor genes important in a variety of cancers and the neurological disorder, hereditary neuralgic amyotrophy $(2,3,6-9)$. 


\section{Benchmarks}
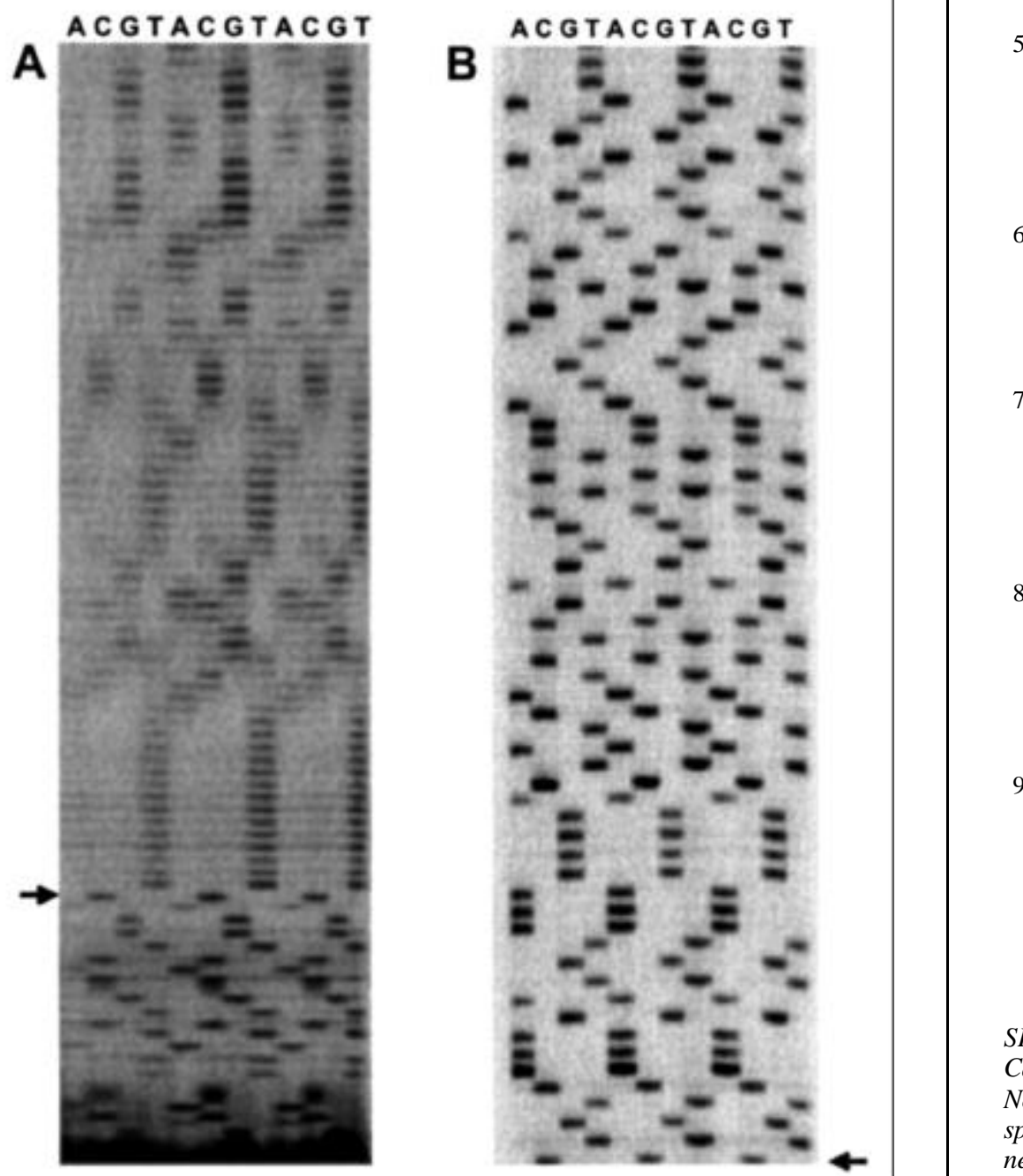

Figure 2. Examples of sequencing gels. (A) Legible sequence obtained up to the poly(A) site $[\operatorname{poly}(\mathrm{T})$ in this figure is due to the use of a reverse primer] that deteriorates in quality and legibility beyond this point $(\rightarrow)$. (B) High-quality sequence data ( $>99 \%$ correct base calling) produced $3^{\prime}$ to the poly(A) site after priming beyond this sequence feature $(\leftarrow)$. The same template DNA was used in both parts A and B.

Our success in this venture indicates that research groups interested in specific areas of the genome should seriously consider performing their own alignments of the sequence data from their regions of interest in comparison with published or locally produced physical maps. We believe that accurate, indepth, local physical mapping data should be used to assist in the alignment of sequence data because it often provides additional, useful positional information for clone fragments. In addition, groups with a vested interest in specific sequence breaks should endeavor to close their own gaps because, in our experience, this can be achieved quickly, even in a small laboratory, using sequence walking techniques.

\section{REFERENCES}

1.Bentley, D.R. 2000. The Human Genome Project—an overview. Med. Res. Rev. 20:189-196.

2.Dunn, J., J. Garde, K. Dolan, J.R. Gosney, R. Sutton, S.J. Meltzer, and J.K. Field. 1999. Multiple target sites of allelic imbalance on chromosome 17 in Barrett's esophageal cancer. Oncogene 18:987-993.

3.Fukino, K., A. Iida, A. Teramoto, G. Sakamoto, F. Kasumi, Y. Nakamura, and M. Eni. 1999. Frequent allelic loss at the TOC locus on $17 \mathrm{q} 25.1$ in primary breast cancers. Genes Chromosomes Cancer 24:345-350.

4.International Human Genome Sequencing Consortium. 2001. Initial sequencing and analysis of the human genome. Nature 409: 860-921.

5.Kuhlenbaumer, G., A. Schirmacher, J. Meuleman, F. Tissir, J. Del-Favero, F. Stogbauer, P. Young, B. Ringelstein, et al. 1999. A sequence-ready BAC/PAC contig and partial transcript map of approximately $1.5 \mathrm{Mb}$ in human chromosome $17 \mathrm{q} 25$ comprising multiple disease genes. Genomics 62:242-250.

6.Meuleman, J., G. Kuhlenbaumer, A. Schirmacher, M. Wehnert, P. De Jonghe, E. De Vriendt, P. Young, E. Airaksinen, et al. 1999. Genetic refinement of the hereditary neuralgic amyotrophy (HNA) locus at chromosome 17q25. Eur. J. Hum. Genet. 7:920-927.

7.Risk, J.M., C. Ruhrberg, H.-C. Hennies, H.S. Mills, T. Di Colandrea, K.E. Evans, A. Ellis, F.M. Watt, et al. 1999 Envoplakin, a possible candidate gene for focal NEPPK/ esophageal cancer (TOC): the integration of genetic and physical maps of the TOC region on 17q25. Genomics 15:234-242.

8.Russell, S.E.H., M.A. McIlhatton, J.F. Burrows, P.G. Donaghy, S. Chanduloy, E.M. Petty, L.M. Kalikin, S.W. Church, et al. 2000. Isolation and mapping of a human Septin gene to a region on chromosome $17 \mathrm{q}$, com monly deleted in sporadic epithelial ovarian tumors. Cancer Res. 60:4729-4734.

9.von Brevern, M., M.C. Hollstein, J.M. Risk, J. Garde, W.P. Bennett, C.C. Harris, K.-R. Muehlbauer, and J.K. Field. 1998. Loss of heterozygosity in sporadic esophageal tumors in the tylosis esophageal cancer (TOC) gene region of chromosome 17q. Oncogene 17:21012105 .

This work was supported by grant no. SP2384/0201 from the Cancer Research Campaign and grant no. CR496 from the North West Research Fund. Address correspondence to Dr. J.M. Risk, Molecular Genetics and Oncology Group, Department of Clinical Dental Sciences, Research Wing, Daulby Street, The University of Liverpool, Liverpool, L69 3GN UK. e-mail: j.m.risk @ liverpool.ac.uk

Received 20 November 2001; accepted 29 March 2002.

\section{J.E. Langan', L. Rowbottom', T. Liloglou ${ }^{1,2}$, J.K. Field ${ }^{1,2}$, and J.M. Risk ${ }^{1}$ \\ 'The University of Liverpool \\ ${ }^{2}$ Roy Castle International \\ Centre for Lung Cancer \\ Research \\ Liverpool, $U K$}

For reprints of this or any other article, contact Reprints@BioTechniques.com 